levels of healthcare worker satisfaction as reported elsewhere (IAS, 2011). This study has resulted in measurable improvements to the health system including: the development of robust internal and external laboratory quality assurance (OA) systems and an integrated training for health workers on congenital syphilis prevention, treatment and quality assured use of PoC technologies. Use of integrated registers in $\mathrm{MCH}$ for data collection allowed for seamless initiation of the service into ANC. Supply chain systems were developed and enhanced especially in Uganda where syphilis testing was previously not routine. 13131 women in Uganda and 12761 women in Zambia received syphilis testing during the 5 month study period with a significant number of tests successfully carried out by nurse/midwives. Integrating syphilis and HIV supply chains led to reduced days of stock out of HIV test kits due to better ordering practices in some sites and did not negatively impact or integration significantly improved HIV service uptake.

Conclusions In addition to being acceptable, feasible and affordable, the systematic introduction of a PoC diagnostic for syphilis can lead to wider health system improvements and enhanced HIV service uptake in ANC. Wider use of PoC technologies is encouraged.

Abstract S4.2 Table1 Uptake of HIV services in sites with concurrent rapid syphilis testing

\begin{tabular}{llll}
\hline & Baseline & $\begin{array}{l}\text { Rapid syphilis } \\
\text { test study period }\end{array}$ & Result \\
\hline HIV testing & Zambia & & \\
$\begin{array}{l}\text { ARV prophylaxis } \\
\text { Referral to care }\end{array}$ & $1,479(95.5 \%)$ & $11151(97.7 \%)$ & $\left(\chi^{2}=74.75 ; p<0.0001\right)$ \\
and treatment & $977(75 \%)$ & $2,036(100.1 \%)$ & $\left(\chi^{2}=35.56 ; p<0.0001\right)$ \\
& Uganda & $1,721(84.6 \%)$ & $\left(\chi^{2}=60.63 ; p<0.0001\right)$ \\
HIV testing & $6,479(95.6 \%)$ & $11192(96.4 \%)$ & \\
$\begin{array}{l}\text { ARV prophylaxis } \\
\text { Referral to care }\end{array}$ & $570(78.5 \%)$ & $964(83.6 \%)$ & $\left(\chi^{2}=7.01 ; p=0.008\right)$ \\
and treatment & $85(16.9 \%)$ & $118(16.1 \%)$ & $\left(\chi^{2}=7.72 ; p=0.006\right)$ \\
\hline
\end{tabular}

\section{S4.3 IMPLEMENTATION OF RAPID TESTS FOR PRENATAL SYPHILIS SCREENING: OVERCOMING HEALTH SYSTEM CONSTRAINTS}

doi:10.1136/sextrans-2011-050102.16

${ }^{1} \mathrm{P}$ J Garcia, ${ }^{2} \mathrm{R}$ Peeling, ${ }^{2} \mathrm{D}$ Mabey, ${ }^{3} \mathrm{~K}$ K Holmes, The CISNE team (Spanish word for SWAN). 'Universidad Peruana Cayetano Heredia, Lima, Peru; '2London School of Hygiene and Tropical Medicine, London, UK; ${ }^{3}$ University of Washington, Seattle, USA

Background Congenital syphilis and syphilis in pregnancy in Peru persist as important public health issues, and improvement of screening/treatment for pregnant women remains challenging. rapid syphilis testing (RST) allows simple and immediate diagnosis and treatment at a single clinic visit and could increase screening and treatment coverage and thereby reduce the incidence of stillbirth and congenital syphilis and generate in the long term a sustainable cost effective intervention.

Methods We tested the feasibility, performance, impact and costeffectiveness of implementing RST in an underserved urban area at a biggest maternity hospital in Peru and a network of 16 peripheral health centres offering prenatal care in a periurban poor area in Callao-Ventanilla, Peru. RST (integrated with HIV rapid test: the "two for one") were offered at the first prenatal visit (ANC), at delivery and within miscarriage/abortion services.

Results Data from the baseline pre-implementation evaluation revealed limited coverage of screening and treatment services for maternal syphilis and a complex and inefficient system for ANC. RST was started in January 2010. Overall success of implementation was measured by rates of maternal syphilis screening and treatment coverage, partner treatment, and acceptability of RST among providers and patients. Complementary evaluations comparing costeffectiveness of RST against the Rapid Plasma Reagin, and a performance analysis of RST against the "multiple gold test" [Rapid Plasma Reagin + Treponema pallidum particle agglutination assay or fluorescent treponemal antibody absorption] were also simultaneously performed. Attention was paid to the successful development of a system of internal and external quality control for testing and test supplies and the process of dissemination and transfer activities to the Ministry of Health of Peru, through the involvement of both the National Program of STIs and HIV and the Reproductive Health Program. National guidelines have been modified, and recommend the use of both tests, RST and rapid HIV testing in the screening of pregnant women.

Conclusions RST implementation was feasible, successful, acceptable and cost effective. Its introduction catalysed improvements in the quality of care, and by the end of the project it has been introduced in the country as a national policy.

\section{S4.4 PROGRESS IN DEVELOPMENT OF DUAL RAPID SYPHILIS TEST TECHNOLOGY}

doi:10.1136/sextrans-2011-050102.17

\section{L Cox. CDC, Atlanta, USA}

Background Traditionally, most diagnoses of syphilis relies on serology and required testing for patient antibodies against cardiolipin (non-treponemal tests) as well as against treponemal antigens (treponemal tests). These serological tests must be performed in clinical laboratories, require considerable scientific resources and trained technicians to perform them, and test results often are not reported for several days. As a result, control in resource-poor countries has been problematic. Rapid tests provide a platform whereby patient antibodies can be quickly analysed (5-30 $\mathrm{min}$ ) by their binding to immobilised antigens on a nitrocellulose membrane. Another advantage is that patient sera, blood, or plasma can be tested, thus requiring fewer resources and technician time to perform. One of the first rapid tests developed was the Abbot Determine (treponemal test results only) which has served as a useful rapid screening tool in some settings. Other treponemal only rapid tests include the Standard Diagnostics BioLine and the Fujirebio Espline. These rapid tests have a sensivitivity ranging from $92 \%$ to $100 \%$ and specificity ranging from 93.4 to $98.9 \%$ when compared to the TP-PA. The Eurostrip (Euromedi Equipment LTD, W. Harrow, UK), another treponemal only rapid test, was recently evaluated at CDC for a potential field study in Kenya. With 94 archived serum samples, the Eurostrip had a sensitivity of $98.6 \%$ and a specificity of $100 \%$ when compared to the TP-PA.

Methods and results A recent breakthrough in technology allows for modified cardiolipin to be attached to membranes, and the first generation of dual rapid tests were developed in collaboration with CDC: the ChemBio DPP Screen and Confirm (ChemBio Diagnostic Systems, Medford, New York, USA) and the Span Spirolipin (Span Diagnostics Inc., Surat, India). In a study with 1601 archived serum samples, the non-treponemal component of the ChemBio DPP had a sensitivity of $97.3 \%$ and a specificity of $98.6 \%$ when compared to the RPR; the treponemal component had a sensitivity of $97 \%$ and a specificity of $95.5 \%$ when compared to the TP-PA. In a similar study with 376 archived serum samples, the non-treponemal component of the Span Spirolipin had a sensitivity of $96.5 \%$ and a specificity of $97.7 \%$ when compared to the RPR and the treponemal component had a sensitivity of $97.3 \%$ and a specificity of $99.1 \%$ when compared to the TP-PA. These evaluations demonstrate that these dual rapid tests are as sensitive and as specific as traditional RPR and TP-PA tests. Conclusions The emergence of dual rapid tests offers resource-poor countries the opportunity for improved point-of-care diagnostic 
capacity. Span has recently announced that they can market the Spirolipin dual test for $<1$ USD per cassette, which would make these tests affordable in many settings. Future developments in rapid testing include the addition of antigen(s) to also test for HIV infection, offering even more diagnostic options to control these diseases, especially among pregnant women.

\section{S4.5 MATERNAL SYPHILIS TESTING AND TREATMENT TO IMPROVE MATERNAL AND CHILD HEALTH SERVICES: PROGRESS AND CHALLENGES}

doi:10.1136/sextrans-2011-050102.18

\section{Kamb. CDC, Atlanta, Georgia, USA}

Background In 2007, WHO released the document Global Elimination of Congenital Syphilis, Rationale and Strategy for Action, developed in collaboration with a team of expert consultants. The strategy aims to prevent mother to child transmission of syphilis through the strengthening of antenatal care programs to ensure:

Early antenatal care for all women, with universal syphilis screening and prompt treatment of those infected

Treatment of all sexual partners of infected women, promotion of condom use during pregnancy, and counselling of all women on how to prevent infection

All neonates born to RPR-positive mothers are given a single dose of penicillin as prophylactic treatment.

This presentation documents progress in the global elimination initiative thus far, notes challenges towards substantial reduction in congenital syphilis by 2015 , and proposes areas for focus in the next 2 years.

Methods/results Most progress has been made in areas of advocacy and recognition of the public health problem, the emergence of rapid diagnostic tests and (in some areas of the world) integrated intervention initiatives such as those linking prevention of mother to child transmission of HIV and congenital syphilis. Progress has also been made in integration of a handful of critical indicators into existing data collection systems, allowing better monitoring of national and global program progress. Publication of program evaluations has been mixed, with increased reporting on evaluations of implementing testing and treatment strategies, but still limited research on validation of proposed impact targets (eg, use of stillbirth as an indicator of impact). Additionally, specific funding remains limited, although an Investment Case for donors is near completion and some new opportunities for funding integrated services have arisen.

Conclusions Some emerging global issues such recent initiatives and trends in global public health financing may influence future progress. National guidelines on testing strategies are needed to avoid less than optimal or more costly than needed testing strategies. More focused efforts on earlier and higher quality antenatal care will likely support congenital syphilis elimination along with promoting better overall antenatal services.

\section{Symposium 5: Unsolved challenges in women's sexual and genital health

S5.1 THE OTHER VAGINITIDES: UPDATE ON DESQUAMMATIVE
INFLAMMATORY VAGINITIS AND REFRACTORY CANDIDA
VAGINITIS

doi:10.1136/sextrans-2011-050102.19

\section{J D Sobel. Wayne State University School of Medicine, Detroit, USA}

Desquammative Inflammatory Vaginitis (DIV) is now recognised as a not uncommon caused of purulent vaginitis with a profuse discharge accompanying dyspareunia and vaginal burning. Less well recognised are the vestibular and vulvar manifestations of this chronic idiopathic and likely immune mediated inflammatory process which occurs exclusively in Caucasian women usually in the peri-menopausal period. The syndrome must be differentiated from trichomoniasis since both share several clinical manifestations including markedly elevated vaginal $\mathrm{pH}$ and inflammatory cell infiltrate, but several features to be discussed allow correct diagnosis.

DIV is likely precipitated by oestrogen deficiency explaining it's unique epidemiology. Treatment consisting of high potency steroids and other local anti-inflammatory agents results in rapid improvement but cure frequently requires prolonged long term therapy.

A new clinical entity of recurrent vaginitis due either to drug resistant Candida albicans and other Candida species including Candida glabrata will be discussed. These frustrating cases constitute a major therapeutic challenge because of intrinsic or acquired fluconazole resistance and frequent cross resistance to the entire Azole drug class. Therapeutic options are few, moreover, in vitro breakpoints defining Azole drug resistance have not been established. Prolonged exposure to fluconazole appears responsible. Cases will be presented describing several forms of refractory drug resistant vaginal candidiasis.

\section{S5.2 ACTION HEROES OR CASUAL OBSERVERS: VAGINAL MICROFLORA AND STI/HIV RISK}

doi:10.1136/sextrans-2011-050102.20

S Hillier. University of Pittsburgh School of Medicine, Pittsburgh, USA

Some components of the microbial flora of the vagina, most notably lactobacilli, have been linked with decreased rates of bacterial and viral STIs, including HIV, mucopurulent cervicitis and pelvic inflammatory disease. However, the same sexual activity which adversely impacts vaginal colonisation by lactobacilli increases risk of STIs. Therefore, the apparent protective benefits of lactobacilli could reflect co-epidemiology rather than an independent biological benefit of these microorganisms. This presentation will present a summary of the epidemiological data linking vaginal microflora to HIV/STI risk and evidence suggesting that lactobacilli play a causal role in reducing risk of STI/HIV in women.

\section{S5.3 NEW DATA ON PELVIC INFLAMMATORY DISEASE: IMPLICATIONS FOR SCREENING AND MANAGEMENT}

doi:10.1136/sextrans-2011-050102.21

J Ross. British Association for Sexual Health and HIV-BASHH, London, UK

Pelvic inflammatory disease is a common cause of morbidity in young sexually active women, but many of our preventive efforts and management strategies are based on empirical approaches. Central to the implementation of chlamydia screening programmes is an understanding of the risk of PID following chlamydial infection. The recent POPI study helps to inform this risk and also provides information about the role of other potential pathogens. The importance of Mycoplasma genitalium as a cause of PID needs to be clarified, but its implications for testing and treatment are now becoming clearer. Women with PID are often poorly adherent to antimicrobial treatment and recent trials suggest that newer treatment regimens may be easier to take and have the potential to improve outcomes. 